\title{
HERBRAND ANALYZING FUNCTIONS
}

\author{
BY BURTON DREBEN ${ }^{1}$ AND STÅL AANDERAA \\ Communicated by G. W. Mackey, May 1, 1964
}

In [1] it was shown that there is no strong analyzing function for crucial applications of Herbrand's rules of passage, and, hence, that there is no such function for the rule of modus ponens. However, there are weak analyzing functions for these rules, and in parts (a) and (b) of the following theorem two simple ones are specified.

TheOREM. Let $S$ and $T$ be any closed quantificational schemata containing $j$ and $k$ quantifiers respectively. Let $r=j+k$, and let $m=\max (p, q)$, where $j, k, p, q \geqq 1$. Finally, let $\gamma(j, p)$ be the function

$$
j^{p}
$$

and let $\phi(j, k, p, q)$ be the function

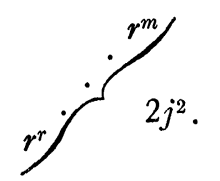

(a) If $S$ has property $C$ of order $p$, and $T$ comes from $S$ by one crucial application of a rule of passage, then $T$ has property $C$ of order $\gamma(j, p)$.

(b) If $S$ has property $C$ of order $p$, and the schema $S \supset T$ has property $C$ of order $q$, then $T$ has property $C$ of order $\phi(j, k, p, q)$.

(c) There is neither a 3-placed function $\delta(j, p, q)$ nor a 3-placed function $\zeta(k, p, q)$ such that whenever $S$ has property $C$ of order $p$ and $S \supset T$ has property $C$ of order $q$, then $T$ has property $C$ either of order $\delta(j, p, q)$ or of order $\zeta(k, p, q)$.

The functions $\gamma$ and $\phi$ do not give the least possible bounds, but they do make clear that the only information needed about the schemata -in addition to property $C$ orders-is the number of quantifiers occurring in the schemata. The argument showing that these functions $\gamma$ and $\phi$ are weak analyzing functions will appear in Dreben's introduction to [2]. It turns on the formula stated at the end of [1]. Here we shall prove part (c) of the theorem by means of examples.

ExAMPLE 1. Let $S$ be the schema

${ }^{1}$ With support from NSF Grant GP-228. 


$$
\left.\left(y_{0}\right)\left[\left(y_{1}\right)\right\rceil M y_{0} y_{1} \vee\left(E x_{0}\right) M y_{0} x_{0}\right] ;
$$

for each $t \geqq 3$, let $A_{t}$ be the schema

$\left(y_{2}\right) \cdots\left(y_{t}\right)\left(E x_{2}\right)\left[\left(G y_{2} x_{2} \& \neg M y_{3} x_{2}\right) \vee\left(G y_{3} x_{2} \& \neg M y_{4} x_{2}\right) \vee \cdots\right.$

$$
\left.\vee\left(G y_{t-1} x_{2} \& \neg M y_{t} x_{2}\right) \vee \neg M y_{2} y_{2} \vee G y_{t} x_{2}\right]
$$

and let $T_{t}$ be the schema

$$
\left.A_{\imath} \vee\left(E x_{1}\right)(E x)\left(M x_{1} x \&(y)\right\rceil G x_{1} y\right)
$$

Now $S$ contains three quantifiers. Moreover, $S$ has property $C$ of order 2 , and $S \supset T_{t}$ has property $C$ of order 4 . But for each $t \geqq 3$, the schema $T_{t}$ has property $C$ of no order earlier than $t+1$. Hence, no function satisfying the conditions on $\delta$ can exist.

Example 2. For each $s \geqq 2$, let $S_{s}$ be the schema

$$
\begin{array}{r}
\left.\left.\left[\left(y_{1}\right)\right\rceil M_{1} y_{1} \vee\left(E x_{1}\right) M_{1} x_{1}\right] \&\left[\left(y_{2}\right)\right\rceil M_{2} y_{2} \vee\left(E x_{2}\right) M_{2} x_{2}\right] \& \cdots \\
\left.\&\left[\left(y_{s}\right)\right\rceil M_{8} y_{s} \vee\left(E x_{s}\right) M_{8} x_{8}\right]
\end{array}
$$

and let $T_{s}$ be the schema

$$
\begin{array}{r}
(E x)(y)\left[\neg M_{1} y \vee M_{s} x \vee\left(M_{1} x \& \neg M_{2} y\right) \vee\left(M_{2} x \& \neg M_{3} y\right) \vee \cdots\right. \\
\left.\vee\left(M_{s-1} x \& \neg M_{s} y\right)\right] .
\end{array}
$$

The schema $T_{s}$ contains just two quantifiers, but has property $C$ of no order earlier than $s+1$. However, the schema $S_{s}$ has property $C$ of order 2, and the schema $S_{s} \supset T_{s}$ has property $C$ of order 3 . So there is no function $\zeta$.

\section{REFERENCES}

1. Burton Dreben, Peter Andrews and Stål Aanderaa, False lemmas in Herbrand, Bull. Amer. Math. Soc. 69 (1963), 699-706.

2. John van Heijenoort, Editor, Jacques Herbrand, Ecrits logiques, Presses Universitaires, Paris.

HARVARD UNIVERSITY 